\title{
Philonsorbonne
}

14 | 2020

Année 2019-2020

\section{Mourir pour des idées : Descartes, le martyr et la règle générale de vérité}

\section{Louis ROUQUAYROL}

\section{Q OpenEdition}

\section{Journals}

Édition électronique

URL : https://journals.openedition.org/philonsorbonne/1439

DOI : 10.4000/philonsorbonne.1439

ISSN : $2270-7336$

Éditeur

Publications de la Sorbonne

\section{Édition imprimée}

Date de publication : 14 février 2020

Pagination : 101-118

ISSN : 1255-183X

\section{Référence électronique}

Louis ROUQUAYROL, " Mourir pour des idées : Descartes, le martyr et la règle générale de vérité »,

Philonsorbonne [En ligne], 14 | 2020, mis en ligne le 26 février 2020, consulté le 08 juin 2021. URL:

http://journals.openedition.org/philonsorbonne/1439; DOI : https://doi.org/10.4000/philonsorbonne. 1439

(c) Tous droits réservés 


\title{
Mourir pour des idées : \\ Descartes, le martyr et la règle générale de vérité
}

\author{
Louis ROUQUAYROL
}

«Aber Blut ist der schlechteste Zeuge der Wahrheit / Le sang est le plus mauvais témoin de la vérité ».

F. Nietzsche,

Ainsi parlait Zarathoustra II, « Des prêtres ».

\section{De l'opiniâtre au fanatique}

En réfléchissant sur la première vérité de la métaphysique, le sujet méditant fait l'hypothèse, au début de la Méditation troisième, que tout ce que nous percevons clairement et distinctement est, par là même, vrai : c'est la règle générale (regula generalis) de vérité ${ }^{1}$. La nécessité de donner à cette règle une validité définitive provoquera autant les recherches sur l'existence et la nature de Dieu (dans la Méditation troisième) que celles sur la possibilité de l'erreur (dans la Méditation quatrième), jusqu'à déboucher sur l'expression de la règle de vérité (regula veritatis) qui permet d'asseoir irrévocablement la possession, par le sujet méditant, d'une science authentique ${ }^{2}$.

1. Ac proinde jam videor pro regula generali posse statuere, illud omne esse verum, quod valde clare et distincte percipio, et par conséquent il me semble déjà que je puis établir pour règle générale que tout cela est vrai que je perçois fort clairement et distinctement. (Meditatio tertia, in Euvres de Descartes, édition de C. Adam et P. Tannery, nouvelle présentation, Paris, Vrin-CNRS, 1964-1974, tome VII, page 35, lignes 13-15. Ci-après: AT, puis la tomaison en chiffres romains, la page et la ligne en chiffres arabes. Sauf lorsque la référence à l'édition française des Méditations est précisée, je traduis le texte latin).

2. La « règle de vérité » (l'expression se trouve dans la Meditatio quinta, AT VII, 70, 1. 26) stipule que « toutes les choses que je perçois clairement et distinctement sont nécessairement vraies », et qu'alors " $\mathrm{j}$ 'en ai une vraie et certaine science (veram et certam de hoc habeo scientiam)» (Meditatio quinta, AT VII, 70, 1. 12-18). 
Qu'une telle règle, qui se trouve au cœur du dispositif argumentatif des Méditations métaphysiques, ait immédiatement donné lieu à de nombreuses objections ne doit dès lors pas étonner. D'ordinaire, on lui oppose deux faits, présentés de façon générale et indéterminée : l'opiniâtreté et l'illusion de la clarté. Ces faits font système. En effet, quelle qu'ait été la force de l'évidence qui nous a attachés à une opinion et quelle que soit la constance de notre assentiment, rien n'indique que cette force suffise à justifier la vérité de notre conception (illusion de clarté), ni que l'assentiment renouvelé soit l'effet de l'évidence elle-même plutôt que d'un mauvais tour pris par notre jugement (opiniâtreté). L'opiniâtreté est ainsi le consentement tenace à une opinion tenue pour certaine à la suite d'une première conception prétendument claire, mais prise pour telle sans réel fondement.

Cette double difficulté s'était présentée d'elle-même à Descartes lorsqu'il formulait pour la première fois dans les Méditations métaphysiques la règle générale de vérité. L'objection prenait alors l'apparence d'un doute rétrospectif formulé par le sujet méditant sur ses anciennes opinions : les choses qui, pour lui, avaient été reçues jusqu'ici comme " très certaines et très manifestes » n'ont-elles pas été rendues " douteuses et incertaines » par le travail de la Méditation première $?^{3}$ En outre, certaines choses ne lui apparaissaient-elles pas «très clairement », qui pourtant ne pouvaient faire l'objet d'une véritable connaissance avant les raisons démonstratives des Méditations elles-mêmes? Sur quoi, dès lors, pouvait se fonder son assurance en ces opinions, si ce n'est sur leur clarté illusoire, ensuite renforcée par une certaine habitude à les croire ? ${ }^{4}$

Certes, la solidité de la saisie par l'esprit de sa propre existence et la nécessité avec laquelle cette dernière est reconnue, dans la Méditation seconde, ne sauraient être confondues avec une opiniâtreté sans fondement. Reste que, au début de la Méditation troisième, si un doute rétrospectif est formulé, c'est dans la mesure où cette solidité et cette nécessité ne suffisent pas encore définitivement à assurer le sujet méditant de l'impossibilité, pour une clarté illusoire, de se faire passer pour une clarté réelle. Autrement dit, rien ne permet d'établir un lien nécessaire et univoque entre la clarté de ma perception, l'assurance de mon assentiment et la vérité de mon jugement, puisque l'opiniâtreté et l'illusion de clarté introduisent dans cette relation l'ambiguiité d'une clarté subjectivement vécue comme suffisante mais en réalité infondée, et la possibilité d'un jugement pris pour vrai qui n'est qu'un préjugé.

3. Verumtamen multa prius ut omnino certa et manifesta admisi, qua tamen postea dubia esse deprehendi, mais pourtant, j'ai admis auparavant beaucoup de choses comme tout à fait certaines et manifestes, lesquelles cependant ensuite ont été suspendues par le doute (Meditatio tertia, AT VII, 35, 1. 16-18).

4. Aliud autem quiddam erat quod affirmabam, quodque etiam ob consuetudinem credendi clare me percipere arbitrabar, mais il y avait encore une autre chose que j'affirmais, qu'à cause d'une habitude que j'avais à la croire je pensais aussi percevoir clairement (loc. cit., 1. 23-26). 
Même lorsqu'elle sera validée par les preuves de l'existence de Dieu et la considération de ses attributs, il semble que l'application de la règle générale de vérité continue de donner lieu à d'inextricables difficultés dans la mesure où elle demeure dépendante du témoignage interne de la conscience, de la présence dans l'esprit d'une perception claire et distincte, et a pu nourrir à ce titre l'idée persistante d'un «subjectivisme" de l'évidence en régime cartésien ${ }^{5}$. Il n'est pas certain que la solution mise en œuvre par Descartes, dans un vif échange avec Hobbes, parvienne à répondre de façon pleinement et définitivement satisfaisante à cette difficulté : distinguer entre l'obstination fondée sur une illusion de clarté et l'authentique évidence ${ }^{6}$ ne revient-il pas à apporter pour solution l'état même de la question? En effet, si la clarté avec laquelle une opinion nous apparaît est, sans aucun doute, la condition nécessaire pour que nous la soutenions avec ténacité sans nous sentir libre d'y donner ou non notre assentiment (notre volonté étant emportée avec une " grande inclination »), il ne s'agit là selon Hobbes que d'une "métaphore » qui ne prouve nullement que de la clarté à la vérité la conséquence soit bonne, pas plus qu'elle ne permet d'attester une différence ferme et assurée entre la clarté et l'illusion de la clarté ${ }^{7}$ Quant à la distinction, pourtant constitutive de l'évidence cartésienne en raison même de la consistance qu'elle apporte à la clarté sous l'espèce d'un protocole intellectuel d'analyse, Hobbes ne la mentionne pas plus ici que Descartes. Sans doute la difficulté soulevée n'est-elle pas étrangère à cet oubli.

Dans les Cinquièmes objections, Gassendi reconduit cette difficulté, donnant ainsi à Descartes une nouvelle occasion de s'expliquer sur les conditions dans lesquelles la distinction entre la clarté et l'illusion de clarté

5. Denis Kambouchner a au contraire insisté sur l'objectivité de la règle générale de vérité, ou plus précisément sur le fait que, dans cette règle, «la demande d'évidence institue l'objectivité elle-même » ("Le statut cartésien de la clarté et de la distinction ", in La clarté à l'âge classique, Paris, Classiques Garnier, 2013, p. 35). Cependant, le reproche constant, fait à Descartes, d'un "subjectivisme», demeure encore à élucider dans une autre de ses dimensions : celle des marques extérieures - objectives, visibles - de cette évidence sur le plan interpersonnel. C'est précisément sur ce plan, comme on va le voir, que Gassendi portera la discussion.

6. Hobbes estime que la clarté dans l'entendement «peut bien être la cause pourquoi quelqu'un aura et défendra avec opiniâtreté quelque opinion (potest ergo lux hacc esse causa quare quis obstinate opinionem aliquam defendat vel teneat), mais elle ne lui peut pas faire connaître avec certitude qu'elle est vraie (sed non quod sciat veram eam esse) » (Troisièmes objections, Objection treizième, AT VII, 192, 1. 3-5 ; IX, 149). Descartes lui répond que cette clarté, tous ne l'ont pas «qui pensent l'avoir (putant se habere) », de sorte qu'elle « diffère beaucoup d'une opinion obstinée (obstinata opinione), qui a été conçue sans une évidente perception » (Troisièmes réponses, loc. cit., 1. 20-23 ; IX, 149).

7. «Cette façon de parler, une grande clarté dans l'entendement, est métaphorique, et partant, n'est pas propre à entrer dans un argument (metaphorica est, nec igitur argumentativa) : or celui qui n'a aucun doute, prétend avoir une semblable clarté, et sa volonté n'a pas une moindre inclination pour affirmer ce dont il n'a aucun doute, que celui qui a une parfaite science (non minorem quam qui revera scit). » (Troisièmes objections, Objection treizième, AT VII, 191, 1. 21 à 192, 1.3 ; IX, 149). 
peut être effective, et de produire les raisons pour lesquelles la règle générale n'est pas vouée à conforter l'opiniâtre dans l'opinion fausse qu'il prétend percevoir clairement. À la différence de Hobbes, cependant, Gassendi prend en apparence en compte la clarté et la distinction pour formuler son scrupule: s'il faut encore, après application de la règle, distinguer entre clarté (et distinction) réelle et clarté (et distinction) illusoire, alors la règle générale est insuffisante et doit être abandonnée.

De plus, Gassendi transpose cette difficulté, déjà présente chez Hobbes, en substituant, à l'opiniâtreté démasquée par l'évolution dans le temps de nos croyances, la confrontation entre des opinions contradictoires soutenues par des personnes différentes à un même moment ${ }^{8}$. La seconde configuration prend ainsi une forme extrême, en ce sens qu'elle suppose une mise à l'épreuve directe de la règle générale de vérité, sans la médiation pacificatrice du temps :

Chacun pense concevoir fort clairement et fort distinctement [l'opinion] qu'il défend; et ne dites point que la plupart ne sont pas fermes dans leurs opinions, ou qu'ils feignent seulement de les bien entendre : car je sais qu'il y en a plusieurs qui les soutiendront au péril de leur vie (et ne dicas plerosque aut harere, aut fingere, sunt ecce, qui pro iis, quas habent, opinionibus etiam mortem oppetant, et ne dites pas que plusieurs hésitent ou feignent qui, pour les opinions qu'ils ont, affronteraient même la mort), quoiqu'ils en voient d'autres portés de la même passion pour l'opinion contraire (tametsi videant alios pro oppositis oppetenteis, quoiqu'ils en voient d'autres qui l'affronteraient pour des opinions opposées); si ce n'est peut-être que vous croyiez que même à ce dernier moment, on déguise encore ses sentiments, et qu'il n'est pas temps de tirer la vérité du plus profond de sa conscience (nisi vero putas tum demum a pectore imo veras voces non ejici, excepté si vous croyez réellement que les vraies pensées ne sortent pas du fond du cœur avant $)^{9}$ ?

Ici, Gassendi prend la ferme intention, voire le fait même de mettre en jeu sa vie pour défendre une opinion, comme le signe de ce qu'elle apparaît à celui qui la conçoit de façon particulièrement (et passionnément) claire et distincte. Au problème de l'opiniâtreté, que s'objectait à lui-même Descartes, s'ajoute désormais l'existence d'un nouveau fait qui déroge à la règle générale de vérité : l'opiniâtreté devient fanatisme, et insidieusement le

\footnotetext{
8. Antonia LoLordo parle d'un " dilemme » posé à Descartes pour déterminer ce qui constitue réellement une perception claire et distincte, ce dilemme pouvant prendre la forme d'un conflit entre différentes opinions «à des moments différents» chez une même personne, ou dans un même temps chez des personnes différentes (" Gassendi as a Critic of Descartes », in The Oxford Handbook of Descartes and Cartesianism, éds. S. Nadler, T. Schmaltz, D. Antoine-Mahut, Oxford, Oxford University Press, 2019, p. 601).

9. Cinquièmes objections, Contre la troisième Méditation, § 1, AT VII, 278, 1. 27 à 279, 1. 1. La traduction est celle de Clerselier dans l'édition française des Méditations métaphysiques de 1647, reproduite dans l'édition des Euvres complètes, dir. J.-M. Beyssade et D. Kambouchner, Paris, Gallimard, «Tel», 2018, volume IV-1, p. 466. Je propose entre parenthèses dans le corps du texte une nouvelle traduction du latin.
} 
fanatisme devient une preuve de la vérité de l'opinion soutenue, puisqu'il ne semble pas qu'il puisse exister de conception plus claire et distincte que celle pour laquelle on accepterait de mourir.

Ce second argument a de surcroît quelque chose d'indiscutablement plus fort que le premier, en raison même de son inscription dans un contexte intersubjectif où des opinions contradictoires sont soutenues : celui qui est intimement persuadé de la clarté et de la distinction de sa conception n'est-il pas autrement coupable de son opiniâtreté lorsqu'il est confronté à la diversité des sentiments? Si l'opiniâtreté quotidienne peut être expliquée par un défaut d'intersubjectivité, soit sous la forme d'une pensée solitaire, soit sous la forme d'un consensus trop grand au sein d'un groupe ${ }^{10}$, elle ne constitue qu'une exception à la règle générale de vérité, exception regrettable mais dont on peut se prévenir en prenant soin d'instruire son jugement par celui des autres. L'opiniâtreté est alors simplement un obstacle à la mise en œuvre du doute (qui constitue l'assise nécessaire à l'obtention d'une perception claire et distincte), et donc à une application légitime de la règle générale de vérité. $\mathrm{Au}$ contraire, plus qu'une exception, l'opiniâtreté fanatique est une objection à la règle générale de vérité : le fanatique reste attaché à son opinion en dépit de cette diversité intellectuelle qui devrait l'amener à douter ${ }^{11}$ et à poursuivre le protocole de l'enquête jusqu'à obtenir une conception réellement claire et distincte. La faiblesse de cette objection apparaîtra plus tard : l'application de l'esprit que suppose la mise en œuvre de la règle générale de vérité est en réalité, chez Descartes, indissociable de ce protocole même ${ }^{12}$.

Reste que, pour autant que cette dimension est momentanément laissée de côté, il s'agit moins pour Gassendi - et c'est là la singularité de son argument - d'opposer au critère cartésien l'expérience en somme banale d'une opiniâtreté surjouée et verbale, que de faire face à la réelle et ferme intention de mourir pour ses idées, et même, au fait de se mettre ainsi en péril pour ses opinions. Qu'il ne soit pas question ici d'une simple intention, c'est ce qu'atteste la fin de notre texte : le latin (tum demum) marque bien, en effet, la dernière extrémité dans laquelle se trouve le fanatique avant de mourir - dernière extrémité rendue dans la traduction de Clerselier par l'idée d'un « dernier moment ». À cette extrémité, quand les épées tirées se

10. L'erreur partagée par tout un groupe acquiert en effet une force de persuasion supérieure. C'est ce qui conduit Descartes à se méfier de l'argument du «consentement universel», puisque «plusieurs [...] peuvent consentir à une même erreur» (À Mersenne, le 16 octobre 1639, AT II, 598, 1. 5-7), et se trouver confortés dans cette opinion à raison de sa popularité.

11. Descartes mentionne comme une raison naturelle de se défaire de ses préjugés le caractère contradictoire des opinions reçues dans une même société ou dans des sociétés différentes : «j'apprenais à ne rien croire trop fermement de ce qui ne m'avait été persuadé que par l'exemple et par la coutume » (Discours de la méthode, Deuxième partie, AT VI, 10, 1. 21-23). Il en va de même pour les opinions contradictoires reçues tout au long de notre enfance.

12. Sur cette dimension, fondamentale, cf. D. Kambouchner, «Remarques sur la définition cartésienne de la clarté et de la distinction », in Les Facultés de l'âme à l'âge classique, dir. C. Jaquet et T. Pavlovits, Paris, Publications de la Sorbonne, 2006, p. 118-119. 
rencontrent sans qu'aucune place ne soit laissée à la feinte, ce qui se trouve au « fond du cœur » ne peut pas ne pas être révélé ; le fait tout intérieur de l'épreuve de la règle générale ne saurait alors manquer, en quelque sorte, de s'extérioriser, de se manifester phénoménalement.

Gassendi fait ainsi subir à cette règle une manière d'expérience cruciale, une contre-épreuve décisive, un rigoureux passage à la limite : si, au moment de mourir, deux hommes qui s'affrontent embrassent des opinions contradictoires qu'ils pensent concevoir chacun de façon fort claire et distincte (et qu'ils ne peuvent pas ne pas concevoir ainsi, étant donné qu'ils sont sur le point de mourir); si, la vérité étant une, une telle situation suppose que l'un des deux se trompe nécessairement ${ }^{13}$; alors la règle générale de vérité est insuffisante pour départager les adversaires, c'est-àdire, en dernier ressort, pour distinguer le vrai d'avec le faux et le douteux (ce qui est précisément sa fonction). Nous sommes au cœur de la difficulté, et l'un des deux adversaires va tragiquement mourir dans une fausse persuasion que ne sera pas parvenue à dissiper la règle générale de vérité.

Du moins est-ce ainsi que Gassendi, en ne donnant pas un rôle positif à la distinction, pourtant constitutive de la règle générale de vérité, parvient à donner à son scrupule quelque chose de saisissant: par la profondeur de l'expérience existentielle qu'il objecte au critère cartésien, il semble satisfaire à l'extrême à l'appel de Descartes à une forme d'intériorité ${ }^{14}$ - à défaut d'être attentif, comme cela apparaîtra dans un instant, aux conditions dans lesquelles cette clarté est effective. On y verra cependant au moins le signe que Gassendi a saisi à la fois l'importance du recours cartésien au témoignage interne de la conscience, et la difficulté d'un tel recours ${ }^{15}$, ce qui

13. Sed quotiescumque duorum de eadem re judicia in contrarias partes feruntur, certum est alterutrum saltem decipi, mais, à chaque fois que deux personnes soutiennent à propos d'une même chose des jugements contraires, il est certain que l'un des deux au moins se trompe (Regula II, AT X, 363, 1. 8-10, je traduis). Pour pouvoir formuler son argument, il était naturel que Gassendi acceptât également cette prémisse. Cf. Disquisitio metaphysica, seu Dubitationes et Instantice adversus Renati Cartesii Metaphysicam et Responsa, texte établi, traduit et annoté par B. Rochot, Paris, Vrin, 1962 (désormais : Instances, puis le numéro de la page dans cette édition) : «car enfin il faut que les choses les plus contraires et même les contradictoires soient vraies (ut plane contraria, imo contradictoria sint vera), si tout ce que chacun juge perçu par lui clairement et distinctement est pleinement vrai » (Doute I, Instance II, op. cit., p. 208-209).

14. Eudoxe déclare dans La Recherche de la Vérité que l'on n'est persuadé de certaines choses « que par sa propre expérience, et par cette conscience ou ce témoignage interne que chacun expérimente en soi-même lorsqu'il considère les choses (quam propria experientia, eaque conscientia, vel interno testimonio, quod in se ipso unusquisque, cum res perpendit, experitur) » (AT X, 524, 1. 14-17).

15. «Note here that Gassendi has not simply brought up the notorious problem of the criterion against Descartes. Rather, he has provided an argument for there being particular need for a criterion in this case, by pointing out that we have all experienced cases where our seemingly clear and distinct perceptions are incompatible with each other or with those of other people. » (A. LoLordo, " "Descartes's One Rule of Logic": Gassendi's Critique of the Doctrine of Clear and Distinct Perception ", British Journal for the History of Philosophy, 13, 1, 2005, p. 57). 
constituera deux bonnes raisons de prendre au sérieux une telle objection. Du moins le scrupule du chanoine de Digne a-t-il le mérite de radicaliser et, par là, de poser de façon parfaitement lisible, ce qui sera caractérisé plus tard comme le (faux ?) problème du subjectivisme de la règle générale de vérité.

\section{Le fanatique congédié}

Descartes dispose pourtant autrement de l'objection, en des termes si simples qu'ils semblent clore définitivement le débat. Quoiqu'il indique d'abord que les objections proposées contre la règle générale de vérité sont les plus sérieuses de Gassendi, Descartes donne ensuite l'impression, comme souvent, de se tirer du piège tendu par son contradicteur en n'acceptant pas les prémisses de son objection :

Ce que vous alléguez ensuite [...] ne prouve rien (sed nihil probans), non plus que ce que vous dites qu'il y a des personnes qui mourraient pour la défense de leurs fausses opinions (ut neque quod quidam pro falsis opinionibus mortem oppetant, non plus que ceux qui affrontent la mort pour des opinions fausses), parce qu'on ne saurait prouver qu'ils conçoivent clairement et distinctement ce qu'ils assurent avec autant d'opiniâtreté (quia probari nunquam potest illos clare et distincte percipere id quod pertinaciter affirmant, parce qu'on ne saurait jamais prouver que ceux-ci perçoivent clairement et distinctement ce qu'ils affirment obstinément) ${ }^{16}$.

Descartes affirme donc avec une certaine désinvolture que l'objection de Gassendi ne porte pas, et semble ne pas faire droit à la complexité du scrupule. Car en alléguant que celui qui a une opinion fausse, et meurt pour celle-ci, ne saurait prouver par là même que celle-ci était claire et distincte, Descartes n'a pas l'air de dire autre chose que ceci : celui qui a une opinion fausse a une opinion fausse (puisque la vérité suppose une perception claire et distincte), quand bien même il serait sur le point de mourir pour elle.

Cependant, à examiner de plus près ce qui semble constituer une tautologie, on observera dans la réponse un glissement ${ }^{17}$ qui n'a rien d'anecdotique : il ne s'agit plus ici de savoir si celui qui défend une opinion au péril de sa vie ne disposerait pas là d'une preuve de la vérité de cette dernière ; il est désormais question, partant du principe que cette opinion est fausse, de déterminer si l'intention de mourir pour elle est une « preuve » de

16. Cinquièmes réponses, AT VII, 361, 1. 18-22. Et, plus haut: «courage : enfin vous apportez ici contre moi quelque raison, ce que je n'ai point remarqué que vous ayez fait jusques ici. » (AT VII, 361, 1. 7-8).

17. Que Gassendi a également remarqué : «vous ajoutez : et le fait que certains recherchent la mort pour de fausses opinions (j'avais dit, moi, pour des opinions contraires) » (Gassendi, Instances, op. cit, p. 208-209). Gassendi ajoute, déçu par la réponse de Descartes : «mais c'est encore un moyen de vous échapper (sed hoc quoque effugium quam praclarum est) ». 
sa clarté (quand bien même elle ne serait pas vraie), ce que Gassendi acceptait tacitement. Si c'est le cas, la règle générale de vérité est également en péril, mais pas à la façon dont l'entendait l'auteur des Cinquièmes objections : non parce qu'une opinion claire et distincte pourrait être vraie ou fausse, de telle sorte que la règle générale de vérité pourrait nous faire prendre le faux pour le vrai ${ }^{18}$, mais parce qu'une opinion fausse pourrait être clairement et distinctement perçue.

L'argument ainsi formulé peut être abordé de deux manières.

Tout d'abord, le glissement textuel qui exprime le refus par Descartes de prendre l'argument par sa pointe (l'abîme de la mort ouvrant à l'alternative $\mathrm{du}$ vrai et du faux) dessine en creux une thèse forte, mais qui demeure inexprimée : pour défendre le contenu d'une croyance, rien ne sert de faire valoir son mode de présentation, ses qualités extrinsèques ou son décorum ${ }^{19}$, fût-il particulièrement impressionnant et, en l'occurrence, mortel. La tautologie de Descartes est donc plus particulièrement instructive par ce qu'elle exclut que par ce qu'elle affirme: elle clôt le champ de la connaissance sur lui-même, et le ferme à l'extériorité (du décorum, comme précédemment de l'autorité des autres hommes ${ }^{20}$ ). Autrement dit, rien ne pourra s'ajouter d'extérieur à notre connaissance qui soit susceptible de la soutenir: la force de la lutte, par exemple, n'apporte rien à une opinion qui serait à même de la rendre vraie ou, semble-t-il, fausse.

Par ailleurs, cette inversion change de fond en comble le sens du problème: à l'ouverture laissée par Gassendi quant à la détermination de la vérité ou de la fausseté de l'opinion considérée, Descartes oppose une situation d'emblée faussée, pour pouvoir plus aisément répondre en distinguant la clarté de l'illusion de clarté. En effet, celui qui meurt pour une opinion fausse ne peut « prouver» que sa conception est réellement claire et distincte. Pour cette raison, et comme avec Hobbes, Descartes n'est-il pas en train de proposer derechef pour réponse l'état de la question, dans la mesure où la possibilité pour une opinion fausse d'être effectivement claire et distincte n'est ni sérieusement examinée ni détruite?

Ce serait le cas si la signification de la preuve réclamée était laissée indéterminée. Certes, quand Descartes affirme qu'on ne saurait «prouver » que l'idée à laquelle le fanatique tient jusqu'à la mort est claire et distincte, on pourrait s'attendre à ce qu'il propose en contrepartie des «marques » de

18. C'est le fond du scrupule de Gassendi, à savoir que cette règle «peut si facilement nous faire recevoir le faux pour le vrai (proclive adeo est falsum pro vero admittere) » (AT VII, 279, 1. 13-14). Comme il le précise dans les Instances : «je dis qu'ils pensent percevoir une chose qu'ils croient vraie, bien qu'elle soit fausse, avec autant de clarté et de distinction que ceux qui ne se trompent pas » (ibid., p. 208-209).

19. Cicéron traduit le prepon de Panétius par decorum : il signifie selon lui l'aspect visible de la vertu (Cicéron, De Officiis, I, 93-95). Comme nous le verrons (§ 4), il s'agit ici précisément du problème de l'aspect sous lequel la règle générale de vérité peut être rendue visible - donc, de son decorum.

20. Juste avant le point qui nous intéresse, Descartes déclare qu'il «ne s'émeut pas beaucoup » de « l'autorité d'autrui » (AT VII, 361, 1. 14-18). 
l'évidence en laquelle doit consister la clarté et la distinction. On ne manquera pas, dans un premier temps, d'être déçu ${ }^{21}$. Cependant, que Descartes n'ait pas assumé positivement cette tâche ne signifie pas qu'il l'ait tout à fait éludée : le témoignage interne de la conscience quant à la clarté et la distinction d'une idée suppose un rigoureux protocole de résistance au doute, succédant à une remise en cause non moins rigoureuse de tous nos préjugés. C'est d'ailleurs ce que Descartes indique dans sa réponse à Gassendi, comme pour donner la signification de cette " preuve » que l'opiniâtre est incapable d'administrer : il ne faut pas se contenter de faire appel à la règle de vérité, mais il faut encore donner les vrais moyens de ne pas se tromper, "premièrement en ôtant les préjugés, puis après en expliquant toutes les principales idées, et enfin en distinguant les claires et distinctes de celles qui sont obscures et confuses $»^{22}$.

Malheureusement, puisque ces critères sont exclusivement éprouvés dans l'intimité de l'examen par chacun de ses propres conceptions, ils sont évidemment insuffisants du point de vue externe - et virtuellement échouent à répondre au cour de l'objection de Gassendi, qui se place précisément à ce point de vue. Pas plus qu'il ne faut soumettre son raisonnement au syllogisme qui contraint par la force de la structure dans la vacance de l'esprit ${ }^{23}$, pas plus la force, qui fait combattre et mourir pour une idée, ne doit impressionner l'esprit au moment d'instruire son jugement. Nul témoignage extérieur, donc, ne peut se substituer au témoignage interne, et la possibilité d'une mise à l'épreuve intersubjective de la règle générale de vérité est, en première analyse, délaissée. C'est ce que regrette Gassendi, dans la mesure où, selon lui, il ne nous est pas possible de pénétrer dans l'esprit de quelqu'un d'autre :

Pour nous, qui sommes des hommes et qui, comme il convient à des hommes, raisonnons d'après ce qui se produit en effet (ex effectis ratiocinamur), c'est une preuve qui nous semble évidente (et qu'assurément vous ne devez pas écarter) qu'ils perçoivent clairement et distinctement une

\footnotetext{
21. Déception dont le sens inaugural a été fixé par Leibniz : «j’ai signalé ailleurs la médiocre utilité de la règle tant vantée (jactatae illius regulae), qu'il ne faut admettre que les connaissances claires et distinctes, tant qu'on n'a pas apporté de meilleures marques (nisi meliores afferantur notae) du clair et du distinct, que celles proposées par Descartes » (Animadversiones in partem generalem Principiorum Cartesianorum, in Die Philosophische Schriften, éd. Gerhardt, Bd. IV, p. 363, trad. P. Schrecker, Paris, Vrin, 2001, p. 60-61, traduction légèrement modifiée).
}

22. Descartes déclare donc à Gassendi avoir donné la «bonne méthode » en son lieu, «ubi primum abstuli omnia prajudicia, et postea enumeravi omnes pracipuas ideas, ac distinxi claras ab obscuris aut confusis » (AT VII, 361, 1.23 à 362, 1.4). En effet, il faut ne « comprendre rien de plus en mes jugements, que ce qui se présenterait si clairement et si distinctement à mon esprit, que je n'eusse aucune occasion de le mettre en doute » (Discours de la méthode, Deuxième partie, AT VI, 18, 1. 19-23, je souligne).

23. Formam... qua vi suce dispositionis concludit, une forme qui conclut par la force de sa structure (Leibniz, Animadversiones, op. cit., p. 62-63). Pour l'opposition de Descartes à tout procédé de ce genre, cf. la Règle $X$ selon laquelle les syllogismes des dialecticiens mettent notre raison en congé (AT X, 405-406). 
certaine opinion comme la meilleure et comme celle qu'il faut suivre, que le fait d'affronter la mort pour elle (quod pro ea mortem oppetant $)^{24}$.

Descartes, pour ce qui regarde la recherche de la vérité, ne semble pas se fonder sur les effets, ou sur des expériences concrètes qui donneraient des signes extérieurs susceptibles d'être reconnus et partagés par des hommes en chair et en os. De quels hommes s'agit-il cependant, dans l'objection de Gassendi ? Il faut encore donner à cette expérience concrète invoquée par le chanoine de Digne toute sa profondeur historique et, avec cela, philosophique, pour éprouver jusqu'au bout la résistance de la philosophie cartésienne.

\section{Du fanatique au martyr}

Descartes ne s'émeut donc guère du scrupule de Gassendi, et dissipe son apparente profondeur. Loin d'être une expérience valide, capable de pouvoir troubler la trouée de la lumière naturelle, l'expérience vague et inconstante de l'opiniâtreté qui s'ignore superbement ou du fanatisme qui erre sont disqualifiées au nom d'une autre forme d'expérience plus élaborée, éduquée par la méthode et faisant de l'évidence le résidu du protocole du doute. Si tel vécu ne vaut pas tel autre, si telle expérience peut certifier l'assurance de son évidence et pas telle autre, il n'en reste pas moins que les marques qui permettent d'en attester ne sont qu'esquissées par Descartes, et demeurent enfermées dans l'examen intérieur, par chacun, de ses propres conceptions.

Cependant, soit que Descartes ait feint de ne pas l'apercevoir, soit que la discussion proprement philosophique ait oblitéré la dimension virtuellement historique de l'argument proposé par Gassendi, le cœur du problème a en fait été éludé. Reprenant en charge la difficulté dans la continuité des scrupules du chanoine de Digne, c'est l'anonyme Hyperaspistes qui introduit cette nouvelle dimension. Sans doute Descartes s'interdit-il de ferrailler sur le terrain épineux de la controverse théologique; toujours est-il que l'Hyperaspistes s'émeut du peu d'attention accordé à l'expérience cruciale de Gassendi, notamment sur son versant religieux.

De plus, je vous le demande, touchant ces personnes que vous dites [...] être prêtes de mourir pour la défense de leurs fausses opinions dont elles ne voient pas clairement la vérité (quarum veritatem perspicue non vident), pensez-vous qu'elles soient de pire condition (peioris conditionis) que les autres, qui souffrent la mort pour de vraies (qui pro veris mortem subeunt), dont toutefois ils ne voient pas plus clairement la vérité, que ceux-là celle de leurs fausses opinions (quarum tamen veritatem non magis, quam primi suarum opinionum falsarum, vident, dont toutefois ils ne voient pas plus la vérité que

24. Instances, op. cit., p. 208-209. 
les premiers ne voient la fausseté de leurs opinions) ? Car ayant dit auparavant que la probabilité suffit pour la conduite de la vie, et les uns et les autres croyant voir cette probabilité, pourquoi la mort et les mérites ne seront-il pas égaux (cur dispares erunt obitus et merita, pourquoi la mort et les mérites seraient-il inégaux) ? Ce qui toutefois est absurde, autrement un hérétique aura autant de mérite dans le martyre qu'un orthodoxe (in martyrio quispiam cum Orthodoxo Horreticus) $)^{25}$.

On le voit, l'Hyperaspistes réalise un double coup de force: il réintroduit le problème, a priori évincé, du rapport entre opiniâtreté et vérité, tout en acceptant la réponse de Descartes à l'objection de Gassendi, puisque la situation théorique est bien celle d'une opinion dont la clarté est mal aperçue ; ce faisant, il donne à la difficulté toute sa profondeur historique et théologique, obligeant Descartes à répondre en chrétien sur le terrain de la religion et, plus généralement, de la conduite de la vie.

Ainsi, pour l'authentique martyr (qui à sa manière fait preuve d'opiniâtreté), il ne semble pas que le rapport à la vérité puisse être esquivé ; il semble même en quelque sorte constitutif de son acte, pour autant que celui-ci témoigne de la vérité de sa foi. Chez Thomas d'Aquin, le martyre est par exemple l'acte par lequel «quelqu'un demeure ferme dans la vérité et la justice contre les assauts de ses persécuteurs $»^{26}$. Le martyr est précisément celui qui, jusqu'au moment ultime, n'abandonne ni la vérité ni la justice. Mais, en réalité, il ne s'agit pas seulement de cela : car si le martyr persiste dans la vérité, il (dé)montre également celle-ci par sa persévérance, ou du moins la manifeste en produisant un témoignage extérieur ${ }^{27}$ qui a valeur de confirmation empirique.

Si le martyre suppose donc comme sa cause la vérité de la foi, on en déduit qu'au contraire l'hérétique peut toujours mourir pour une opinion, y compris du reste pour une vérité, mais que sa mort n'étant pas animée par la foi, il n'aura pas rang de martyr. Réciproquement, la défense de toute vérité - et même d'une vérité scientifique - pour autant qu'elle s'arrime au refus du mensonge (contraire à la loi de Dieu) donne droit au titre de martyr ${ }^{28}$. À

25. [L'Hyperaspistes] à Descartes, juillet 1641 (§ 3), AT III, 401, 1. 18-28.

26. Pertinet autem ad rationem martyrii ut aliquis firmiter stet in veritate et iustitia contra persequentium impetus. (Summa theologiae, Iia IIae, q. 124, a. 1, c.).

27. «Parce qu'on montre (ostenditur) d'autant plus qu'on aime quelque chose [en l'occurrence, la charité] que, pour elle, on méprise ce qu'on aime le plus [sc. : la vie] et on choisit de souffrir la chose la plus odieuse » (q. 124, a. 3, c.). Ainsi, « les martyrs sont appelés pour ainsi dire des témoins (martyres dicuntur quasi testes) parce que, par leurs souffrances corporelles qu'ils endurent jusqu'à la mort, ils rendent témoignage à la vérité (quia scilicet corporalibus suis passionibus usque ad mortem testimonium perhibent veritati)» (a. 5, c.).

28. À l'objection selon laquelle «si l'on mourait pour avoir confessé la vérité de la géométrie ou de toute autre science spéculative, on serait martyr, ce qui semble ridicule (si quis moreretur pro confessione veritatis geometriae, vel alterius scientiae speculativae, esset martyr, quod videtur ridiculum)»(a. 5, obj. 2), Thomas répond que "parce que tout mensonge est un péché, comme nous l'avons vu (q. 110, art. 3 et 4), le désir d'éviter le mensonge, en tant que le mensonge est contraire à la loi de Dieu, peut être une cause de 
cet égard, le martyr peut être le témoin d'une vérité particulière, dont la défense est animée par le témoignage d'une vérité plus haute, la vérité de la foi. Soutenir une vérité particulière, scientifique, n'est pas directement la cause du martyre - mais l'est indirectement, pourvu que sa connaissance soit attisée par une vérité plus haute et le refus du mensonge.

Ce rapport indirect est impliqué dans la première difficulté soulevée par l'Hyperaspistes, pour lequel il s'agit d'éprouver la règle générale de vérité à l'aune d'une réalité d'ordre supérieur : la foi. En effet, si la vérité de la foi ne peut jamais être clairement aperçue par le fidèle, pas plus clairement en tout cas que l'hérétique ne perçoit sa propre erreur ou ce qu'il pense être la vérité, il en résulte que la règle générale est à nouveau rendue inopérante pour distinguer entre l'authentique vérité et l'erreur de l'hérétique. Quel critère proposera alors Descartes pour distinguer celui qui meurt pour la vérité, quand bien même elle serait insuffisamment perçue, et l'hérétique qui, ne percevant pas plus clairement son opinion, est néanmoins dans le faux ?

Face à un problème d'une telle ampleur, Descartes n'est-il pas enfin contraint de reconnaître le lien étroit entre la force (puisque le martyre est un acte de force) et la vérité, tout en laissant de côté la règle générale, manifestement insuffisante?

À cette difficulté, la réponse de Descartes est double, et consiste à montrer que l'objection est à la fois un hors sujet, et la redite d'une objection déjà formulée et publiquement réfutée :

Et je n'ai pas dit que par la lumière de la grâce, nous connaissions clairement les mystères de foi (encore que je ne nie pas que cela ne se puisse faire), mais seulement que nous avions confiance qu'il les faut croire (nos confidere illis esse credendum). Or personne ne peut trouver étrange, s'il est vraiment fidèle, et ne peut même douter qu'il ne soit très évident, qu'il faut croire (esse credenda) les choses que Dieu a révélées, et qu'il ne faille préférer les lumières de la grâce à celle de la nature (nemini fidem catholicam vere habenti dubium, et personne de foi catholique ne peut vraiment en douter). Et tout ce que vous me demandez ensuite ne me regarde point, puisque je n'ai donné aucune occasion en mes écrits de me faire de telles demandes. Et parce que j'ai déjà ci-devant déclaré en ma réponse aux Sixièmes objections, que je ne répondrai point à de telles questions, je n'ajouterai ici rien davantage ${ }^{29}$.

D'une part, donc, il faut reconnaître à la lumière de la grâce une puissance spécifique, de sorte que le fidèle peut percevoir confusément la vérité de son contenu mais percevoir en même temps clairement qu'il

martyre, quelle que soit la vérité qu'il blesse (quia omne mendacium peccatum est, ut supra habitum est, vitatio mendacii, contra quamcumque veritatem sit, inquantum mendacium est peccatum divinae legi contrarium, potest esse martyrii causa)» (a. 5, resp. 2).

29. À [l'Hyperaspistes], août 1641, AT III, 426, 1. 2-13. On retrouve dans ce texte les deux travers que déplore généralement Descartes dans les objections de l'Hyperaspistes, et qui l'amènent à ne pas les publier avec les Meditationes : " je dois considérer l'intérêt du lecteur, lequel s'ennuierait de voir des redites, ou des choses qui sont hors de sujet» (À Mersenne, 22 juillet 1641, AT III, 417). 
doit croire ce dernier ${ }^{30}$. Cependant, quoique cette lumière puisse être dite intérieure $^{31}$, il n'est pas question ici de faire appel à la règle générale de vérité qui n'a de validité que pour la lumière naturelle, et ne peut s'accommoder d'une matière obscure - quand bien même elle serait éclairée d'une volonté extraordinaire d'y acquiescer (ou plutôt, justement parce qu'une telle lumière se suffit amplement à elle-même). Et de fait, c'est d'un autre type de certitude qu'il s'agit, si bien qu'en toute vraie foi, la règle générale de vérité qui vaut pour la lumière naturelle doit se démettre, dans certains cas, face à une sorte de règle générale de la foi qui vaut pour la lumière surnaturelle. Cette règle générale consistera, pour l'essentiel, dans une sincère adhésion du croyant à ce qui dépasse l'entendement, dans la mesure où il s'agit de choses révélées.

Cette concession étant faite, il n'y a plus lieu de relier le problème du martyr à celui de la vérité telle qu'elle se manifeste dans la règle générale et par la lumière naturelle : sur ce second point, il n'y aura donc qu'une fin de non-recevoir. En mêlant les registres de la recherche de la vérité et de la conduite de la vie, de la science et de la religion, l'Hyperaspistes lui-même a prêté le flanc à la réplique de Descartes. Dans les Méditations, il n'a été question que de la lumière naturelle, étant mis à part tout ce qui relève de la lumière surnaturelle et du témoignage de la foi ${ }^{32}$. Le problème du martyre relève de cet autre domaine dans lequel Descartes ne s'aventure pas et, de ce fait, est expulsé du champ de la vérité profane.

À nouveau, le silence de Descartes est sans doute plus significatif que son refus: il n'est plus question, comme c'était par exemple le cas chez Thomas d'Aquin, d'affirmer que l'on peut mourir en martyr pour une vérité scientifique, et témoigner par là d'une vérité plus haute (la défense de toute vérité pouvant être animée par la foi). Il n'est plus question que le règne de la grâce parachève celui de la nature, ni que la vérité de la foi couronne la vérité scientifique dans un acte de force; il s'agit, au contraire, de bien séparer ces règnes, et de renvoyer ces vérités chacune à leur spécificité ${ }^{33}$.

30. « Car encore qu'on dise que la foi a pour objet des choses obscures (fides dicatur esse de obscuris), néanmoins ce pour quoi nous les croyons (illud, propter quod ipsam amplectimur) n'est pas obscur, mais il est plus clair qu'aucune lumière naturelle » (Secondes réponses, AT VII, 147, 1. 15-17 et IX, 115).

31. En effet, cette « raison formelle » par laquelle nous croyons, dans la foi, est « une certaine lumière intérieure (lumine quodam interno), de laquelle Dieu nous [a] surnaturellement éclairés (quo a Deo supernaturaliter illustrati)» (Secondes réponses, AT VII, 148, 1. 7-13 et IX, 116).

32. Descartes écrit à propos de la théologie : «je ne sens point en moi d'inspiration divine (nec tantum in me divina gratice experior), qui me fasse juger capable de l'enseigner. C'est pourquoi je fais ici ma déclaration, que désormais je ne répondrai plus à de pareilles objections » (Sixièmes réponses, AT VII, 429, 1. 6-8; IX, 230).

33. En ce sens, Descartes ne tombe pas non plus dans la facilité qui consisterait à confondre la vérité scientifique et la véracité d'un témoignage religieux. À propos d'un célèbre texte de Pascal (« Je ne crois que les histoires dont les témoins se feraient égorger», Brunschvicg 593 / Lafuma 822 / Sellier 663), Henri Gouhier écrivait en effet qu'il serait « trop facile », faisant cette confusion, « de constater que le fait de mourir pour une idée n'a rien à voir avec la vérité de cette idée » (Blaise Pascal : commentaires, Paris, Vrin, 2005, deuxième édition, p. 228). 


\section{Du martyr au sage}

«Tout ce que vous me demandez ensuite ne me regarde point»: en résistant à l'insistance de l'Hyperaspistes, Descartes affirme donc tacitement que pour défendre une vérité issue des sciences humaines, il n'est pas nécessaire d'être animé par la foi - dont la lumière surnaturelle ne répond pas au canon de la règle générale de vérité. Sans se prononcer par ailleurs sur l'opportunité morale de mourir ou non pour une opinion religieuse, Descartes peut donc reconduire une nouvelle fois la réponse faite à Hobbes, puis à Gassendi. Dès lors, la difficulté qu'une telle réponse enveloppait semble persister : il faut malheureusement s'en tenir au seul témoignage interne de notre conscience, à notre propre perception, à l'intimité d'une évidence invisible d'un point de vue intersubjectif. Certes, ce témoignage, cette perception et cette évidence sont soutenus par le doute et la méthode, mais ils demeurent, comme l'écrit Gassendi, impénétrables "pour nous, qui sommes des hommes » et qui «raisonnons d'après ce qui se produit en effet » dans le monde.

À moins d'établir que l'objection ainsi formulée n'a aucun sens en contexte cartésien, il reste cependant à examiner, pour répondre à l'incrédulité de Gassendi, si la règle générale de vérité ne serait pas susceptible chez Descartes de prendre figure humaine et, loin de se limiter au seul témoignage interne de la conscience, de s'incarner. Car s'il est évident que l'Hyperaspistes et Gassendi n'ont pas été attentifs, dans leurs objections, aux conditions dans lesquelles la règle générale de vérité doit être mise en œuvre (valeur de la distinction, exclusion de la conduite de la vie et de la foi), il n'en reste pas moins que, pour eux, une incompréhension insurpassable demeure à raison de la difficulté qu'il y a à produire des marques extérieures de cette règle. Qu'il ne s'agisse pas là, du moins explicitement, du problème tel qu'il se pose à Descartes, n'empêche pas la difficulté de subsister - et de constituer pour partie un argument en direction du « subjectivisme » cartésien -, pas plus qu'elle n'empêche d'en chercher, chez Descartes même, la solution.

On sera surpris, d'abord, de retrouver sous la plume de Descartes la figure du martyr. Par une étrange inversion, celui qui est par ailleurs peu enclin à s'enthousiasmer pour les histoires de martyrs ${ }^{34}$ semble n'accepter désormais pour tels que ceux qui sont persécutés pour sa philosophie, c'està-dire pour la vérité elle-même. Aussi Regius endosse-t-il régulièrement, au cours de la « querelle d'Utrecht », le rôle du martyr officiel de la philosophie

34. À propos du récit d'une jeune femme qui imite « les douleurs des martyrs », Descartes remarque par exemple : " on en dit encore plusieurs autres extravagances, mais je crois que celles-ci suffisent pour vous obliger à n'en rien croire. Outre que je connais celui que le Père Mersenne allègue pour son auteur, et je le juge si digne de foi que s'il m'avait dit que ceux de la Chine ont communément deux yeux comme nous, je croirais que la Chine est un pays où il n’y a que des borgnes ou des Cyclopes » (À Huygens, le 12 mars 1640, AT III, 746). 
cartésienne ${ }^{35}$. Plutôt que d'une prétention sérieuse à constituer une martyrologie des sectateurs de la philosophie nouvelle, il s'agit en quelque sorte d'une transposition ironique de thèmes religieux. L'idée, appuyée par une référence scripturaire ${ }^{36}$, selon laquelle il serait courageux de souffrir pour la vérité par probité ou innocence ne constitue dès lors pas à proprement parler une thèse. Pour ce qui relève de la raison purement humaine, et comme cela a été établi, le martyr ne saurait rien prouver de la vérité d'une opinion: que la souffrance pour la défense d'une telle vérité soit moralement louable ne change rien à cette situation.

C'est plutôt vers une seconde figure qu'il faut se tourner, dont l'apparition est aussi soudaine que déterminante, et qui entretient une relation privilégiée avec la règle générale de vérité. Car non seulement Descartes détruit les prétentions du martyr à délivrer des marques extérieures d'une conception vraie par sa mort, mais il substitue en outre à cette figure un autre personnage, dont il y a lieu de penser que le comportement est également caractérisé, quoique d'une façon diamétralement opposée.

En effet, à Bourdin qui réitère contre la règle générale de vérité les objections déjà inlassablement répétées, Descartes propose la figure du sage comme celle qui permettra, sur le plan de l'expérience, de distinguer définitivement entre l'illusion de clarté et la clarté véritable.

Il est bien vrai qu'il en eût pu conclure [ $s c$. : à partir de la règle générale de vérité] que tout ce qui est clairement et distinctement conçu par quelqu'un est vrai, encore que celui-là cependant puisse douter s'il dort, ou s'il veille ; ou même aussi, si l'on veut, encore qu'il dorme, ou qu'il ne soit pas en son bon sens (quamvis somniet, quamvis sit delirus, qu'il dorme, ou qu'il soit extravagant); pource que rien ne peut être clairement et distinctement conçu par qui que ce soit, qu'il ne soit tel qu'il le conçoit, c'est-à-dire qu'il ne soit vrai. Mais pource qu'il n'appartient qu'aux personnes sages de distinguer entre ce qui est clairement conçu, et ce qui semble et paraît seulement l'être (soli prudentes recte distinguunt inter id quod ita percipitur, et id quod tantum videtur vel apparet), je ne m'étonne pas que ce bon homme prenne ici l'un pour l'autre ${ }^{37}$.

35. Ce sont les usages les plus significatifs du terme «martyr » dans l'œuvre de Descartes. Ainsi le «pauvre Mr de Roy, qui sera le premier martyr de ma Philosophie » (À Huygens, le 20 septembre 1643, AT IV, 750). Ou encore : «Monsieur Regius, qu'on pensait enseigner mes opinions touchant la Philosophie, et qui a été en hasard d'en être le premier Martyr» (Lettre apologétique aux magistrats d'Utrecht, AT VIII-2, 208, 1. 5-9, in La Querelle d'Utrecht, éd. T. Verbeek, Paris, Les impressions nouvelles, 1988, p. 410).

36. «Je vous félicite de la persécution que vous souffrez pour la vérité (gratulor tibi, quod persecutionem patiaris propter veritatem) 》 (̇̀ Regius, mars 1642, AT III, 536, 1. 2-3). La citation biblique est la suivante : « Heureux ceux qui sont persécutés pour la justice (beati qui persecutionem patiuntur propter justitiam), car le royaume des cieux est à eux » (Matthieu, 5:10).

37. Septièmes réponses, AT VII, 461, 1. 21 à 462, 1. 4. La dispute tourne autour de l'idée selon laquelle « quand bien même je dormirais (quamvis somniarem), tout ce qui se présente à mon esprit avec évidence, est absolument véritable » (Meditatio quinta, AT VII, 71, 1. 1-2 ; IX, 56). Après la regula generalis de la Meditatio tertia et la regula veritatis de la Meditatio 
La règle générale de vérité devient ainsi, dans sa mise en œuvre, l'apanage d'un sujet particulier: l'homme sage ou prudent. Contrairement au sage, le fou comme le martyr ne sont pas en mesure de distinguer effectivement entre la clarté véritable et l'illusion de clarté. Certes, l'insensé peut découvrir une vérité, mais seulement par accident, c'est-à-dire sans être capable d'affirmer positivement qu'il est en possession d'une perception claire, et distinguée d'une perception confuse. C'est pourquoi seul le sage dont il reste encore à élucider les prérogatives ${ }^{38}$ - possède la vérité et la distingue nettement de l'erreur; seule la persuasion de celui-ci est définitivement solide et consciente de ses attendus ${ }^{39}$.

Car au-delà de cette caractérisation négative de la sagesse, et sans qu'il soit possible de poursuivre ici très avant la description de la figure du sage, on peut supposer que la règle générale ne prendra chair - c'est-à-dire : ne deviendra visible - que chez un être qui, d'une part, fait profession de se soumettre ingénument à ce que lui indique la raison, et qui, d'autre part, se conduit au mieux dans sa vie, avec mesure et discernement ${ }^{40}$.

La difficulté est-elle pour autant résolue ? Ne pourrait-il pas y avoir deux sortes de sagesse, l'une pour la conduite ordinaire de l'existence et l'autre pour la recherche de la vérité, de telle sorte que celui qui se comporte comme un sage pourrait très bien par ailleurs être déraisonnable sur le plan spéculatif, et réciproquement? La possession de la vérité est-elle nécessairement liée à la manifestation de qualités morales assignables et visibles; la sagesse qui fait usage de la règle générale s'atteste-t-elle dans des marques extérieures?

L'objection, en réalité, n'aura pas de sens pour la philosophie cartésienne.

Il faut ici distinguer deux cas. Descartes reconnaît que ceux qui sont ordinairement considérés comme philosophes, et qui prétendent aimer la

quinta, cette réponse à l'objection de Bourdin esquisse donc une nouvelle et ultime étape dans la construction de la règle de vérité, en précisant qui est à même de la mettre correctement en œuvre.

38. Elles ne sauraient l'être de façon approfondie dans les limites de cet article. Pour une caractérisation au moins sommaire des prérogatives du sage chez Descartes, cf. infra, note 44.

39. Comme chez les Stoïciens, l'enfant ou le fou peuvent dire des choses vraies, mais seul le sage possède, au sens strict, la vérité (Sextus Empiricus, Esquisses pyrrhoniennes, II, 81-83). La science appartient au sage dans la mesure où elle n'est pas autre chose qu'une vertu logique, qui consiste « à savoir si l'on dit vrai ou faux " (J.-B. Gourinat, La Dialectique des stö̈ciens, Paris, Vrin, 2000, p. 60). Voyez également : Cicéron, Premiers Académiques, II, 57, mais aussi Eustache de Saint-Paul, pour qui l'appréhension « claire et distincte [...] se trouve chez les sages (in sapientibus)» (Summa Philosophice Quadripartita I, 24, cité par Étienne Gilson dans l'Index scolastico-cartésien, $\mathrm{n}^{\circ}$ 148, Paris, Vrin, 1979, p. 86).

40. Le sage a en effet une « volonté ferme et agissante d'user toujours droitement, autant qu'il peut, de sa raison, et d'accomplir tout ce qu'il connaît comme le meilleur (quisquis enim firmam et efficacem habet voluntatem recte semper utendi sua ratione, quantum in se est, idque omne quod optimum esse cognoscit exsequendi, revera sapiens est) " (Epistola dedicatoria des Principia, AT VIII-1, 2, 1. 25-28, traduction de D. Moreau, Paris, Vrin, 2009, p. 61). 
sagesse, ne se comportent pas de façon très prudente: ainsi, ils sont " souvent moins sages et moins raisonnables que d'autres qui ne se sont jamais appliqués à cette étude ${ }^{41}$. Il semble alors que la prétendue sagesse $\mathrm{du}$ philosophe soit compatible avec un comportement confinant à l'extravagance (par exemple celui du sceptique).

Ce cas de figure est, en toute rigueur, impossible chez Descartes. En effet, cette extravagance n'est possible que dans la mesure où les anciens philosophes n'étaient pas parfaitement sages, au sens où ils n'apercevaient pas clairement la vérité et n'avaient pas conçu les véritables principes. Si la sagesse consiste dans la découverte de vérités sur lesquelles tous les esprits doivent s'accorder rationnellement, cet accord se manifestera à coup sûr de façon notable, de la même manière que les conceptions confuses de la philosophie vulgaire se remarquent dans le désaccord, et finalement dans la dispute. Il n'est donc pas certain qu'il faille établir une distinction définitive entre la recherche de la vérité et la conduite de la vie. La philosophie cartésienne manifeste ainsi sa vérité d'une façon à la fois visible, sensible et concrète :

les vérités qu'ils [sc. : les Principes] contiennent étant très claires, et très certaines, ôteront tous sujets de dispute, et ainsi disposeront les esprits à la douceur et à la concorde : tout au contraire des controverses de l'École, qui, rendant insensiblement ceux qui les apprennent plus pointilleux et plus opiniâtres, sont peut-être la première cause des hérésies et des dissensions qui travaillent maintenant le monde ${ }^{42}$.

Le caractère contradictoire des opinions soutenues par les philosophes est le signe du manque de clarté et de distinction de leurs principes, et par conséquent une cause de discorde dans la société. Au contraire, la vérité une fois clairement et distinctement conçue doit conduire à un accord, et le sage qui la possède est idéalement à même de convaincre celui qui ne la possède pas $^{43}$. A la situation agonistique proposée par Gassendi pour défaire la règle générale, la philosophie cartésienne est en mesure de substituer une relation pédagogique d'enseignement pour la confirmer.

Parce qu'elle est la prérogative du sage, l'utilisation de la règle générale de vérité suppose ainsi la possession d'un certain nombre de qualités morales, à commencer par une soumission sincère de la volonté à ce que l'entendement aperçoit clairement ${ }^{44}$. Face à la mauvaise foi des

41. Lettre-Préface des Principes de la philosophie, AT IX-2, 4, 1. 27-31.

42. Lettre-Préface, AT IX-2, 18, 1. 8-16.

43. Si enim hujus ratio esset certa et evidens, ita illam alteri posset proponere, ut ejus etiam intellectum tandem convinceret, si, en effet, celui-ci avait une raison certaine et évidente, alors il pourrait la proposer à l'autre de telle sorte que l'entendement de celui-là serait, en fin de compte, convaincu. (Regula II, AT X, 363, 1. 11-13).

44. Selon Geneviève Rodis-Lewis, « la spéculation philosophique exige en effet le respect de la vérité, la probité et la sincérité » en raison du rôle de la volonté dans le jugement (L'Individualité selon Descartes, Paris, Vrin, 1950, p. 130). C'est ce que la Lettre à Dinet nomme la veritatis reverentia, et probitas, et candor (AT VII, 565, 1. 14-15). 
contradicteurs, la vérité ne s'imposera jamais par des moyens violents ; plutôt grâce à une certaine prudence dans sa communication ${ }^{45}$. On comprend désormais que le fait de mourir pour une idée n'est pas une preuve de la clarté de celle-ci, dans la mesure où la reconnaissance de cette clarté (et de sa distinction avec la clarté illusoire) n'est pas le privilège du martyr, mais du sage qui, seul et de façon objectivement constatable, se soumet sans détour à l'évidence. La règle générale de vérité, parce qu'elle s'incarne dans le sage, ne saurait conduire au fanatisme et, d'une certaine manière, tout comportement fanatique prouvera par les effets que l'opinion qui y conduit n'est pas conçue de façon parfaitement claire.

À l'opiniâtreté, Descartes opposait, on s'en souvient, la médiation pacificatrice du temps, qui permet à chacun de remettre en question ses anciennes croyances par le protocole du doute, jusqu'à s'accorder avec sa nature rationnelle sous la dictée de la lumière naturelle; au martyr, tout se passe comme si Descartes opposait à nouveau la méditation du temps, celui de l'enseignement et de la communication d'une vérité portée par la sagesse, qui permettent de produire l'accord des esprits entre eux. Quant à mourir pour des idées, « le sage en hésitant tourne autour du tombeau » ${ }^{46}$.

45. La prudence suppose parfois de persuader «insensiblement» les autres (À Mersenne, le 28 janvier 1641, AT III, 298, 1. 1-7), parfois « de se taire » en attendant des temps meilleurs (À Regius, fin juillet-début août 1645, AT IV , 256, 1. 15-16), parfois de recourir à une autorité extra-philosophique (Meditationes, Epistola, AT VII, 6, 1. 5-9; IX, 8).

46. S'il faut, dans la chanson, mourir pour des idées « de mort lente », c'est précisément parce qu'il est aisé de se « tromper d'idée », et qu'entre plusieurs, tant qu'il n'a pas trouvé la bonne, le sage ne saurait se montrer opiniâtre. Le problème du sacrifice de soi, tel qu'il est posé par Descartes (notamment dans la Lettre à Élisabeth du 15 septembre 1645, AT IV, 293), diffère de celui abordé dans cette étude, puisqu'il ne s'agit pas d'y penser le rapport entre vérité et martyr. 